\title{
Dutch Pendulum Observations in a Submarine with a New Apparatus.
}

$\mathrm{A}^{\mathrm{T}}$ the request of the Dutch Geodetic Committee, the Minister of Marine has once more allowed Dr. Vening Meinesz to make pendulum observations on board a submarine of the Royal Navy, bound for Java, on the voyage from Helder to Alexandria. ${ }^{1}$ He left Helder on October I5 on board H.M. Submarine $\mathrm{K}$ XI, and arrived at Alexandria on November I2, after having touched at Sevilla and Tunis. On November 23 he embarked at Port Said on board the Dutch mail-steamer Koningin der Nederlanden and disembarked on December 5 at Amsterdam.

The main object of the voyage was the trial of a new pendulum apparatus constructed on the lines described by Dr. Vening Meinesz in No. 5, Janvier/ Mars I925, of the Bulletin Géodésique, the organ of the geodetic section of the Geodetic and Geophysic Union. With the consent of the Minister of "Waterstaat" and the director in chief of the Royal Meteorological Institute at de Bilt, Prof. Dr. E. van Everdingen, the apparatus was constructed in the mechanical workshop of this Institute by the chief instrument-maker, Mr. L. L. M. van Rest, with the co-operation of the assistant director, Dr. C. Schoute. ${ }^{2}$

A detailed description of the apparatus with the necessary illustrations will appear in a publication of the Geodetic Committee; for the time being the following cursory description may suffice.

The whole apparatus consists of three parts: the support with the pendulums, the suspension apparatus and the recording apparatus.

The support contains three quarter-metre pendulums of the common Von Sterneck model, the same as were used on the first voyage. These are of brass, not of bronze as was stated erroneously in my former communication.

The three pendulums are rendered as near as possible isochronous. They oscillate in the same plane and can be set going simultaneously with an exactly adjustable amplitude. The oscillations are recorded on a moving photographic film by the light reflected by a mirror fixed to the pendulum, but not, as with the former apparatus, for each of these separately. The light-ray reaches first the mirror of the first pendulum; next that of the second. It records in this way the difference of the elongation angles. The result is that the film gives the curve of a hypothetical pendulum, of which the revolving. vector is the difference of the vectors of the constituent pendulums. As the disturbances of those, caused by the horizontal accelerations due to the movement of the vessel, are the same, their effect is eliminated from the hypothetical pendulum.

In the same way, a second hypothetical pendulum is recorded by the third pendulum in combination with the second. The two curves give two independent results, which control one another and increase the accuracy.

1 Particulars of the voyage on board H.M. Submarine K II from Helder to Batavia in 1923 are given in NATURE of September 15 and December I, I923, and March I and May 3, I924.

2 If geodetic offices in other countries should wish to purchase a similar pparatus, the "Nederlandsche Seintoestellenfabriek" at Hilversum will undertake the construction.

NO. 2945, VOL. I I 7]

For the amplitude reductions of the observations, the movement of the second pendulum must be recorded separately; the ray which performs this is on its way reflected by a small, highly damped pendulum, of a construction devised by Dr. Vening Meinesz, the plane of oscillation of which is parallel to that of the other pendulum. In this way the movement of the middle pendulum is recorded with respect to the vertical.

A second highly damped pendulum of the same construction, the plane of oscillation of which is perpendicular to the plane of the others, serves to record the inclination of this plane during the observations. It was controlled by a small undamped pendulum, but the results proved that this control is not necessary.

The changes of temperature within the apparatus

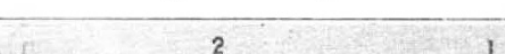


carries the pendulums, the fixed mirrors and prisms and the two lenses. The three agate planes on which the knife-edges of the pendulums rest lie in exactly the same plane; they have been ground and adjusted by the firm Hahn in Cassel (Germany). The base plate rests on three foot screws; parallel to the plane of oscillation of the pendulums a small level is affixed; the bubble is, during the observations, in constant movement owing to the rolling of the vessel; the pendulums must be set free the moment that the bubble is at zero, in order to begin the oscillations with the desired amplitude. Between the top and the base plate are placed a dummy pendulum with thermometer and a hair hygrometer.

The support is encased in a metallic cover with double walls, resting on the base plate; the interstice is filled with a bad conductor of heat. A receiver is not needed. The time of an observation being about thirty-five minutes, it is not necessary to operate in a vacuum, which would cause a great complication. In the cover are three windows: one for each of the film moves past a vertical slit in the front wall of the box, $9 \mathrm{~mm}$. wide and $\mathrm{I} 2 \mathrm{~cm}$. high ; through this pass the rays coming from the support. The lenses form images of the light-slit on the film, but by a cylindrical lens placed before the recording-slit they are reduced to points. The distance between the optical centres of the lenses is the same as that between the light-slit and the recording-slit. As both slits and both lenses are placed symmetrically with regard to the suspension axis, the place of an image of the light-slit on the film is not changed by the relative movements of the free hanging support and the steady recording apparatus which are caused by the rolling of the vessel.

By the movement of the film the bundles of rays describe the different curves, which, owing to the periodical movements of the shutters, show small gaps that indicate the time elapsed.

The distance between the support and the recording apparatus is $\mathrm{I} .03$ metre; a tube of blackened cardboard joins the second lens and the vertical slit, and prevents undesired light striking the film.

The two chronometers, constructed by Nardin at Locle, Switzerland, are excellent timekeepers; the irregularities of the daily rate do not exceed $\mathrm{O} \cdot \mathrm{I}$ sec. One, giving sidereal time, has been in use since I903, the other, giving mean time, is new. It was judged useful to have two chronometers for the purpose of checking each other.

It may be said that in practice the apparatus has surpassed our expectations. The curves recorded on the film are perfectly clear and distinct; their appearance is wholly different from that shown by the films obtained in r923. This may appear from the accompanying specimens (Figs. I and 2) when compared with those given in the Geographical Journal for June I 925. The amplitudes of the hypothetical pendulums are entirely regular, which proves that the Mediterranean Sea, submerged.
Undamped pendulum $\mathrm{D}^{\prime}$ absent.

two lenses and one for reading the thermometer and the hygrometer.

The whole is placed in the suspension apparatus, the plane of oscillation of the pendulums perpendicular to the suspension axis. The latter must be parallel to the keel of the vessel ; in this way the effect of the rolling is neutralised. Originally the apparatus was suspended from pivots, but as the friction caused sudden jerks, these were replaced during the stay at Sevilla by knives, which proved most satisfactory.

The recording apparatus contains two compartments: the small light box and the larger film box.

Behind the light box is placed a Wolfram arc-lamp the rays of this enter the box through a hole in the back. In the front wall is the small horizontal lightslit, $0.2 \mathrm{~mm}$. wide and $5.0 \mathrm{~mm}$. long, through which the rays leave the box. The interior contains the apparatus for the electromagnetic movement of two shutters, governed by a pair of chronometers, each of which every half second prevents for a moment the light-rays from passing the slit.

The film box contains two vertical cylinders, one bearing the film, which by a clockwork is gradually wound round the other. By the displacement of a lever the velocity with which the film moves can be made $\mathrm{I} \cdot \mathrm{O}$ or $0.33 \mathrm{~mm}$. per second; the first is used at the beginning and the end of the observations, the latter in the intermediate time. Between the cylinders the influence of the horizontal accelerations is wholly eliminated. Letting alone the slow decrease caused by the damping, the amplitudes do not vary more than $0.1 \mathrm{~mm}$., that is 2 to 3 per cent. of the whole amplitude. The vertical accelerations are only perceptible in the track of the half-second gaps, which show slight fluctuations; by tracing a straight line through a number of them the influence is easily eliminated.

Sliding of the pendulums is only to be feared in peculiar circumstances. Thanks to the suspension apparatus, the rolling has no influence, but when the pitching exceeds a few degrees, sliding is noticeable, and the images may even altogether disappear from the film. By the use of the horizontal rudder the pitching of a submerged submarine can generally be greatly reduced, if necessary, after submerging to a greater depth.

Observations with the suspending apparatus hanging free or fastened, executed in quiet water, proved that there is no systematic influence caused by this apparatus.

The advantages of the new apparatus over the old one may be summarised as follows :

r. The exertion required from the observer is a great deal less; he is not compelled during the observations to keep the body in a cramped posture under a black cloth, such as covered the old apparatus. 
2. The measurement of the films is very easy, thanks to the clearness and regularity of the recorded curves.

3. The computations are simplified; as the films directly give the movement of the hypothetical pendulums, these have not to be derived by computation from the curves for each separate pendulum.

4. The accuracy of the observations is greater; it is not inferior to that of observations on land.

5. The apparatus may still be used in circumstances where the old one would be useless.

As to the accuracy of the results, it may be remarked that two circumstances unconnected with the apparatus make it somewhat less than that obtainable on land. The first is the varying rate of the chronometers, which may differ during the observations from the mean value, deduced from the timesignals. If scientific radio time-signals of very high precision could be obtained every hour, this would meet the difficulty. The influence is lessened by the use of two chronometers.

The second circumstance is the displacement of the vessel during the observations by the sea currents and the resulting contribution to the Eötvös effect. This cannot be determined with accuracy and an average value must be used. I think it improbable that this difficulty will be easily removed.

On board, the apparatus was placed in the central compartment, near the metacentrum, between the two periscopes, one of which was lifted to enable the light-rays to pass.

At the beginning of an observation the first and the third pendulum were set in motion with the desired amplitude. The middle pendulum, hanging free, began to oscillate through the horizontal accelerations caused by the movement of the vessel.

The depth of the sea was found by applying the echo method by means of the underwater clocksignals. The lapse of time between the giving of the signal and the perception of the echo was determined by a stop-watch.

The time-signals used to determine the rates of the chronometers were generally those of the Eiffel Tower, Bordeaux or Nauen. The relative rate of the chronometers during the observations could be deduced with very great accuracy from the half-second gaps on the films. So far as the computations have been executed, they agree fairly well with the mean relative rates deduced from the time-signals.
During the voyage the following observations were made :

October I 7 . Mouth of the Channel, near the French coast.

, I8. Bay of Biscay, not far from the Spanish coast.

, 26. Sevilla, on the Guadalquivir.

, 28, 29, 30. Mediterranean Sea, between Gibraltar and Tunis.

November 2. Harbour of Tunis.

$$
\begin{gathered}
\text { " 8, 9, Io. Mediterranean Sea, between } \\
\text { Malta and Alexandria. } \\
\text { I2. Harbour of Alexandria. }
\end{gathered}
$$

Some of the observations have been provisionally computed; the results for the harbours of Tunis and Alexandria differ respectively by 0.001 and 0.007 $\mathrm{cm}$. $/ \mathrm{sec}^{-2}$ from the values obtained in $\mathbf{r} 923$.

The weather was generally very fine and the sea smooth; but on November 27, after the vessel left the harbour of Tunis, a heavy gale was blowing, which prevented the making of observations. The small number of observations in the Atlantic Ocean must be imputed to the circumstance that the time fixed for the track from Helder to Sevilla allowed but small delay. The observations in the Mediterranean Sea constitute a valuable complement to that executed in I923.

November 26 , on board the mail-steamer Koningin dev Nederlanden, between Crete and Sicily, Dr. Vening Meinesz made a series of observations. The sea was very smooth and the movements of the ship slight. The apparatus was placed in a state-room; practical difficulties prevented the use of the suspension apparatus. Some of the observations had to be repeated because of the sliding of the pendulums. The films show the influence of the vibrations caused by the engines, but the amplitudes are very regular. The weather during the rest of the voyage prevented further experiments being made, but it appears from the results that in favourable circumstances it is possible to use the apparatus on board a mail-steamer.

I think that Dr. Vening Meinesz may be heartily congratulated on the success he has achieved with his apparatus. The Geodetic Committee cherishes a strong hope that he will be enabled in the course of I 926 to use it on a second voyage to Java, but this time through the Panama Canal, in this manner concluding a series of determinations of gravity at sea all around the world.

\section{Life in the Bed of the Sea.}

I $\mathrm{N}$ recent years many attempts have been made to obtain more definite information than the older naturalists had about the composition and the constitution of the collections of animals living in the bed especially of the European shallow seas. It was known in a vague way that certain animals were to be found, for example, in a muddy and others in a sandy bottom at certain depths; hence the challenge to modern naturalists to clarify the condition of our knowledge.

The first serious attempt was made by Allen on the grounds off Plymouth, and it was proved clearly that certain forms were definitely associated in that locality with a certain type of bottom which was defined in terms of the percentage composition in size of the particles composing the ground soil. Later, Petersen, employing an approximately accurate instrument, the

1 Ministry of Agriculture and Fisheries. Fishery Investigations, Series 2, Vol. 8, No. 4, I925: "Quantitative Studies on the Fauna of the Sea Sea, I92I-24." "By F. M. Davis. Pp. 50. (London: H.M. Stationery Sea, I921-24.
Office, 1925.) 5 s. net.

NO. 2945 , VOL. II 7$]$ grab, for taking a definite area of the sea-bottom, obtained figures which showed which animals were really dominant and characteristic in the different areas examined, but that worker failed to follow up Allen's precise work on the physical composition of the soil, and therefore failed to obtain critical information on the problem attacked. Other workers-notably Ford, who attempted to combine the methods of Allen and Petersen-followed, and have in the main always found communities of animals, but communities also in the main in their own particular locality of silty, muddy, sandy, gravelly or stony soil.

It has remained, however, for Davis in a recent publication ${ }^{1}$ to combine to some extent the methods of the two chief investigators. Petersen had found different groups of animals living together in different localities and suggested that such animals lived in communities, which, however, might vary in different geographical but equivalent localities by the dropping out or addition of certain members. The later 